\title{
Explicit finite element method for in-vivo mechanics of abdominal aortic aneurysm
}

\author{
Michelle D. Gasbarro* - Kenji Shimada** \\ Elena S. Di Martino*** \\ * Biomedical Engineering Department, Carnegie Mellon University \\ 5000 Forbes Ave, Pittsburgh, PA, 15213, USA and MDG Solutions, INC. \\ Pittsburgh, PA, 15217, USA \\ mgasbarr@andrew.cmu.edu \\ ** Mechanical Engineering, Biomedical Engineering Departments and Institute \\ for Complex Engineered Systems, Carnegie Mellon University \\ 5000 Forbes Ave, Pittsburgh, PA, 15213, USA \\ shimada@cmu.edu \\ *** Biomedical Engineering Department and Institute for Complex Engineered \\ Systems, Carnegie Mellon University \\ 5000 Forbes Ave, Pittsburgh, PA, 15213, USA \\ elenad@cmu.edu
}

\begin{abstract}
In this paper we present a strongly-coupled, dynamic, fluid structure interaction analysis of the abdominal aorta in the presence of aneurysm, using an explicit finite element method. All dominating features of the biological system under study were taken into account, including blood dynamics and wall mechanics and the interaction between the two. The inclusion of the surrounding organs and structures that are in contact with the infra-renal aortic segment added physiological realism to the simulation and proved to be a good approach to integrate the interaction of the aorta with its environment. A similar solution strategy could be advantageous to the study of other cardiovascular structures that require a strong coupling among fluid, solid and surrounding entity behaviors.

RÉSUMÉ. Cet article présente l'analyse et la simulation numérique de phénomènes d'interaction fluide-structure dans un anévrisme de l'aorte abdominale en utilisant une résolution directe des équations couplées fluide-structure en régime dynamique. Tous les phénomènes plus importants du système biologique ont été considerés, tels que le fluide dynamique du sang, le comportement de la structure et l'interaction entre les deux. L'inclusion des organes abdominaux et de toutes structures en contact avec l'aorte abdominale infrarénale apporte la preuve évidente de l'avantage de l'approche utilisée. Une solution similaire pourrait être utilisée en cas d'autres problèmes cardiovasculaires qui nécessitent un couplage fort entre le fluide, la structure et le comportement des organes alentour.
\end{abstract}

REMN - 16/2007. Fluid structure interaction, pages 337 to 363 
KEYWORDS: explicit method, abdominal aortic aneurysm, computational, fluid structure interaction.

MOTS-CLÉS: algorithme explicite, anévrisme aorte abdominale, simulation numérique, interaction fluide-structure.

DOI:10.3166/REMN.16.337-363@ 2007 Lavoisier, Paris. Tous droits réservés

\section{Glossary}

F

I

$\mathbf{C}=\mathbf{F}^{T} \mathbf{F}$

$\mathbf{B}=\mathbf{F F}^{T}$

$\mathrm{I}_{1}, \mathrm{I}_{2}, \mathrm{I}_{3}$

b

$\rho$

$\mathbf{P}$

$\boldsymbol{\sigma}$

D

$\mathbf{v}$

$\eta$

$\lambda$

$\psi$

$e$

$\mathrm{p}$

E

V deformation gradient tensor

identity matrix

right Cauchy-Green deformation tensor

left Cauchy-Green deformation tensor (Finger tensor)

first and second invariant of the left CauchyGreen deformation tensor

reference body force per unit volume

mass density

First Piola_Kirchhoff stress tensor

Cauchy stress tensor

rate of deformation tensor

velocity vector field

dynamic viscosity coefficient

bulk viscosity coefficient

isothermal strain energy density function

internal energy

pressure field

elastic stiffness

volume 


\section{Introduction}

The mechanical environment of the cardiovascular system has direct implications in maintaining a healthy physiologic state and in the development of cardiovascular pathologies. In fact, altered stresses and strains affect the delicate balance that regulates homeostasis in the cardiovascular organs. As a consequence, many studies have focused on elucidating the true stresses acting upon cardiovascular structures.

Finite element analysis (FEA) has previously been demonstrated as a useful computational tool to study cardiovascular mechanics (Schmid et al., 1995; Fillinger et al., 2002; Steinman et al., 2003). Unlike most man-made mechanical structures, living tissues are inherently complex, due to their non linear, time-varying material properties and the pulsatile nature of blood flow. Specific engineering tools are available to construct virtual geometries of vessel anatomy from the integration of in-vivo imaging and reconstruction techniques (Smith et al., 2000; Redaelli et al., 2002). These realistic geometries together with computational fluid dynamics and stress analyses techniques make it possible to evaluate cardiovascular physiology and pathology in a virtual space that enables us to increase diagnostic accuracy, and explore solutions for improved patient treatment.

From a broad prospective, stress- and fluid-based FEA methods can be broken into three categories, static, transient, and dynamic. Static analyses study the relationships between externally-applied loads on a deformable body and the resulting forces that are generated within the body. Static analyses embody scenarios that do not depend on loading as a function of time. Transient analyses study events that are dominated by short time-spans, or impulse loads, such as abrupt impacts or explosions. Dynamic analyses also serve the purpose of characterizing deformable body response to time-dependent loading, but unlike transient analyses, dynamic analyses represent the class of problems that cover longer time scales, such as random vibration, natural frequencies, or cyclic events.

Explicit FEA methods have capabilities in static, transient, and dynamic analyses, but they are best suited for short frequency timing events such as complex dynamics, wave propagation problems, high-speed impacts, shock and vibration problems, and extreme material and geometrically non-linear responses. Premise for this work was to explore possible advantages of using explicit, dynamic finite element techniques in order to more accurately capture the in-vivo mechanics of abdominal aortic aneurysms.

An abdominal aortic aneurysm (AAA) is defined clinically as a dilatation occurring in the aorta below the diaphragm (Figure 1). The etiology of AAA is still unclear; however there is evidence that the vessel dilatation is a result of tissue weakening stemming from an unbalanced equilibrium between the flow-induced mechanical and hemodynamic forces and the tissue biochemical response (Curci et al., 1998; Huffman et al., 2000; Ailawadi et al., 2003). 
While this investigation does not pursue specific biomechanical data pertaining to the formation or rupture of AAA, AAA was chosen because geometrically-driven flow phenomena dominate the vessel response thus making it a perfect case study for developing this methodology. It has been shown that viscous blood flow through a healthy descending aorta of relatively constant diameter can be characterized as fully-developed, steady flow (Nakamura et al., 2004). However, in the presence of AAA, the flow patterns quickly transition from steady to disturbed at the entrance to the aneurismal sac due to the dramatic changes in vessel diameter and shape (Bluestein et al., 1996; Peattie et al., 2004). As the flowing fluid core passes into the dilated aneurysm, there is emergence of complex vortex patterns within the sac, and subsequent flow spreading and impingements. Inevitably, such flow patterns have profound influence over the stress distributions that generate within the vessel wall. Another reason for selecting AAA as the first case study is the hypothesis that surrounding entities will radically influence the pulsatile behavior of vessels. Clearly, the descending aorta is tightly restrained within the human trunk as a result of contact between it, the spinal column, the peritoneum membrane, and surrounding abdominal cavity contents making it an ideal application for our purposes.

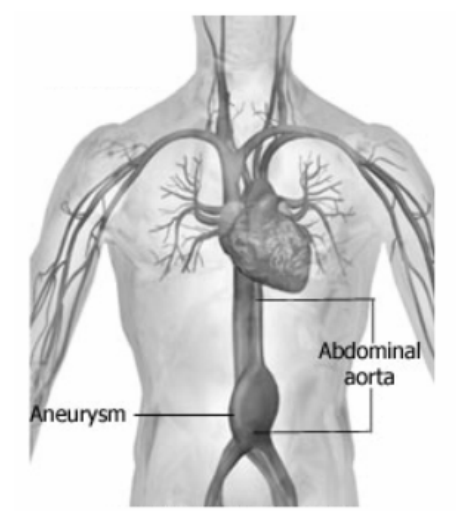

Figure 1. Abdominal aortic aneurysm

Several computational studies of AAA have been reported in the literature. A few published results included the presence of the intraluminal thrombus (ILT), which is often present inside the sac (Harter, 1982; Mower et al., 1997; Di Martino et al., 1998; Wang et al., 2002). In these studies, the most widely used boundary conditions consisted of elimination of rigid body motion by restraining the ends of the artery in all degrees of freedom. The applied load most often was a static pressure corresponding to the peak-systolic phase. (Mower et al., 1997; Vorp et al., 1998; Raghavan et al., 2000; Fillinger et al., 2002). However, a static pressure loading does not represent the actual in-vivo loading condition. Only in recent times, the integral relationship between the blood flow and the arterial wall response was contemplated, coupling 
together the two domains with their corresponding physics. To the authors' knowledge, there have been only two studies regarding FSI in patient-specific AAA (Di Martino et al., 2001; Wolters et al., 2005). Arbitrarian Lagrangian Eulerian schemes have been adopted to solve the coupled interaction between blood and the arterial wall by (Di Martino et al., 2001) and more recently by (Wolters et al., 2005). In both these studies the material properties for the aortic wall were considered linearly elastic and the latter does not include the presence of ILT.

As stated, the first purpose of this study was to capture the all anatomical features that influence the dynamic pulsatile behavior of the abdominal aorta in the presence of aneurysm. To accomplish this, we treated the interaction between the systemic circulation and the vessel that supports the flow as the fundamental forcing function that imposes on the deformable wall thus causing time-varying wall dynamics. In addition, we set the analysis in the domain of large deformation, required by the deformations experienced in vivo by the aorta, and used non linearly elastic material models obtained from experimental data.

\section{Methods}

\subsection{The computational model}

Figure 2 shows the complete FEA model which includes spinal column, retro peritoneum, healthy and aneurysmal aorta segments, ILT, and an abdominal cavity component in the form of a wrapped-around layer whose purpose is to provide the bearing pressure on the aorta due to the abdominal cavity organs. Within these components is the luminal channel, where blood flows. The total length of the aortic vessel is $42 \mathrm{~cm}$. The geometry and finite element mesh were generated using the commercially-available HyperMesh (Altair Engineering, Inc., Troy, MI). The spinal column was obtained from a library of STL files of the human anatomy, while the remaining components originated in HyperMesh. Generalized hypothetical geometries for the aorta and AAA were created with realistic geometrical features, such as the center axis curvature for the aorta due to the presence of the spine. The diameters proximal and midway down the aneurysm sac are consistent with those of a representative $\mathrm{AAA}, 2 \mathrm{~cm}$ and $5 \mathrm{~cm}$, respectively. Also, an adequate length extension was provided at the proximal end of the aorta to allow the emergence of fully-developed flow prior to the aneurysm dilation. Figure 2 a shows the complete geometrical model, while Figure $2 \mathrm{~b}$ shows the retro peritoneum situated between the spinal column and aorta; Figure $2 \mathrm{c}$ shows the aorta when the intestinal component is removed.

A description of all components of the computational model is presented in the following. 


\subsubsection{The aorta}

Based on previous studies, which successfully characterized the differences in mechanical properties between healthy and aneurysmal wall tissue (Vande Geest et al., 2005), the aorta was divided in two segments: the healthy segment which contains the entire aorta distal to the aneurysm sac, and the diseased segment of the aorta. Shell elements were chosen to model the vessel wall, due to the thickness-tosurface area ratio. To capture an accurate through-thickness stress distribution, five integration points through the thickness of the shell elements were defined. The undeformed thicknesses for the healthy and aneurismal segments of aorta are $1.5 \mathrm{~mm}$ and $1.9 \mathrm{~mm}$, respectively, as obtained from averaged excised tissue measurements (Raghavan et al., 2000).

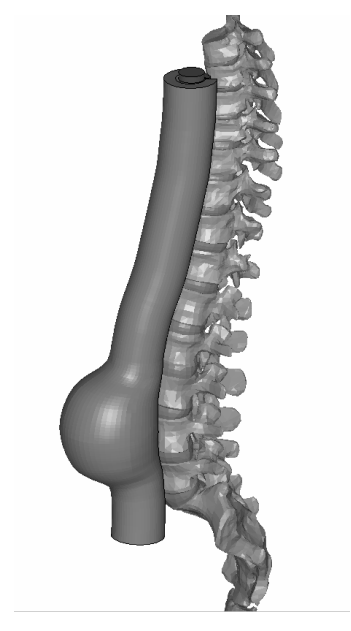

a) intestinal component (outside light grey component)

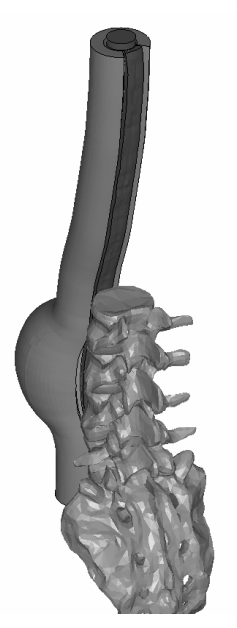

b) retro peritoneum (dark grey)

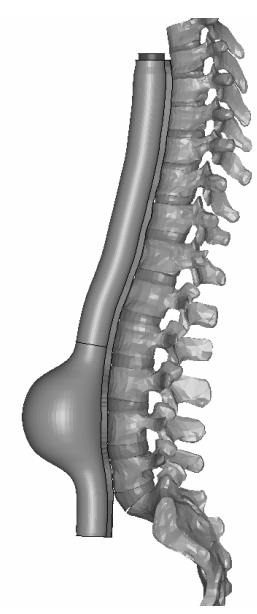

c) abdominal cavity component removed, aorta shown

Figure 2. The computational model: AAA geometry, proximal extension, spinal column, AAA sac, and intestinal component

\subsubsection{The intraluminal thrombus}

A varying thickness inner solid was modeled inside the aneurysmal sac to represent the ILT which lines the inside of an abdominal aortic aneurysm in most patients. Figure 3 shows a viewing plane taken through the center of the aneurysmal sac. The ILT layer has three elements through the thickness in the anterior region and one element in the posterior region. This choice balances the need for anatomical realism, which calls for an asymmetric ILT shape with varying thickness, and the computational time required to run the analysis. At least three solid elements through the thickness are normally used to provide sufficient 
resolution of the through-thickness stress distribution, especially in the presence of bending. However, this would cause the smallest element in the model to be extremely small, which renders an explicit finite element analysis very time consuming. Our choice is justified by observing that the movement of the posterior arterial wall is limited by the spinal column.

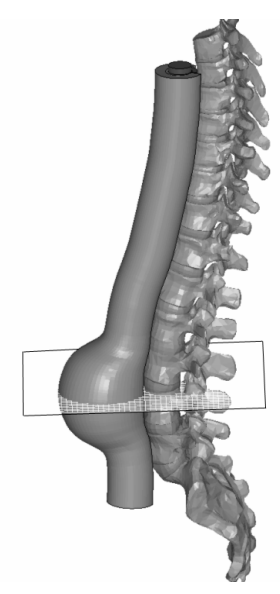

a) viewing plane through aneurysm dilatation

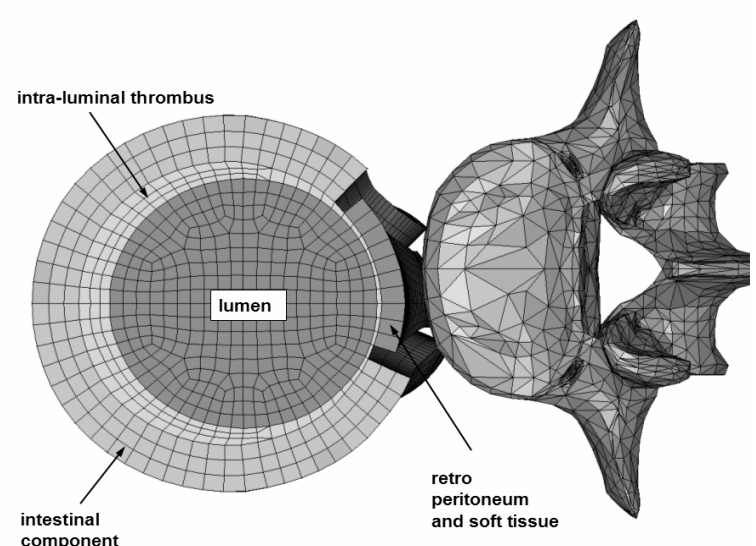

b) mesh detail

Figure 3. Mesh representation of lumen, thrombus, and retro peritoneum membrane

\subsubsection{The blood}

The fluid domain was modeled using eight-nodes single point integration hexahedral elements. The fluid domain consisted of a control volume representing the lumen, and ambient elements positioned at the top of the lumen, which are capable of supplying fluid through specified velocity or pressure conditions. To compensate for only constant state variable capabilities inherent with single point integration solids, a fine mesh density was used throughout the fluid zone to capture flow field and results variability.

2.1.4. The abdominal cavity component, the spine and the retroperitoneum membrane

No experimental data exist that documents what the interface forces are between the abdominal aorta and the surrounding hard and soft tissue entities. The abdominal aorta is enclosed in a cavity covered by an extensive serous membrane, the peritoneum. The organs adjacent to the aorta, including the greater part of the digestive tube, liver, pancreas, spleen and kidneys, are all contained and bound 
together by the peritoneum. Also acting on the aorta is a posterior pressure exerted from the spinal column. In this tight context, there are clear limits over how much dilation of the aorta can occur within each cardiac cycle.

As a first attempt to capture these anatomical boundary conditions, we modeled the tissues surrounding the posterior wall of the aorta as a layer of soft-tissue between the aorta and the spinal column. In addition, there is a layer of four-node shell elements placed between the soft tissue layer and the spinal column, representing the retro peritoneum membrane.

To geometrically represent the abdominal organs and peritoneum, it is assumed that the pulsatile dilation of the aorta is not felt far from the aorta. Moreover, it is assumed that the geometric details of the abdominal organs are not essential to capture their mechanical influence on the aorta. As a consequence, the abdominal cavity component was modeled as a single wrap of material surrounding the aorta except for the posterior side next to the spinal column.

The solid domain includes 97,416 elements, of which 38,070 are hexahedral solid elements defining the intestinal component, the ILT, and the retro peritoneum. The remaining elements are three- and four-nodes shell elements, used for the external surface of the spinal column and for the aortic wall, which follow the Belytschko-Tsay formulation. The fluid domain includes 44,280 hexaedral elements that occupy the length of the lumen channel. The total count for both structural and fluid domains is 141,696 elements.

\subsection{The computational methods}

\subsubsection{Fluid structure interaction method}

To model the interaction between the fluid (blood) and the surrounding structures, we utilized an Arbitrary-Lagrangian-Eulerian (ALE) mathematical description of the field equations in order to have that material domain (Lagrangian) and spatial (Eulerian) domain coexist and are treated by a unified set of field equations. The ALE solver involves a Lagrangian step, during which the mesh is allowed to move and a second step that advects the element state variables back onto a reference mesh. Following this scheme, the fluid domain is updated at each time step to minimize mesh distortion and an advection step is performed at each time step, as described in the following.

\subsubsection{Conservation laws}

Noting that the gravitational forces acting on the system are neglected as well as all other forms of energy excluding mechanical energy, and that isothermal conditions are assumed, the following local conservation equations in a Lagrangian reference are to be solved: 
Conservation of mass

$$
\rho=\frac{\rho_{r}}{J}
$$

Conservation of linear momentum

$$
\rho_{r} \dot{v}_{r}-\operatorname{Div}(\mathbf{P})=\mathbf{b}
$$

where $\rho_{r}$ is the material density in the reference configuration, $\dot{v}_{r}$ is the material acceleration field, $\mathbf{P}$ is the first Piola-Kirchhoff stress tensor, and $\mathbf{b}$ is the reference body force per unit reference volume acting on the region $\Omega$. The unknowns are $\rho(\mathbf{X}, t), \mathbf{v}_{r}(\mathbf{X}, t)$, where $\mathbf{X}$ spans the body in its reference configuration.

We then assume that the region $\Omega$ consists of two parts, a solid region $\Omega_{s}$ and a fluid region $\Omega_{f}$.

In the case of incompressible hyperelastic materials, the Piola Kirchhoff stress tensor is defined as:

$$
\mathbf{P}=-p \mathbf{F}^{-T}+\frac{\partial \psi(\mathbf{F})}{\partial \mathbf{F}} \text { of equivalently } \mathbf{P}=-p \mathbf{F}^{-T}+2 \mathbf{F} \frac{\partial \psi(\mathbf{C})}{\partial \mathbf{C}}
$$

where $\mathrm{p}$ is the hydrostatic pressure, and $\psi$ is the strain energy function, defined as a function of $\mathbf{F}$ or $\mathbf{C}$.

The Cauchy stress tensor $\sigma$ for a Newtonian incompressible fluid can be written as:

$$
\boldsymbol{\sigma}_{f}=-p \mathbf{I}+2 \eta \mathbf{D}
$$

where $p$ is the hydrostatic pressure, $\eta$ is the dynamic viscosity and $\mathbf{D}$ is the rate of deformation tensor:

$$
\mathbf{D}=1 / 2\left[\nabla \mathbf{v}+(\nabla \mathbf{v})^{T}\right] \text { or, in index notation } D_{i j}=1 / 2\left[\frac{\partial v_{i}}{\partial x_{j}}+\frac{\partial v_{j}}{\partial x_{i}}\right]
$$

where $\mathbf{V}$ is the spatial velocity associated with the flow field, related to the material velocity $\mathbf{v}_{r}$ by:

$$
\mathbf{v}_{\mathbf{r}}(t, \mathbf{X})=\mathbf{v}(t, \mathbf{x})
$$

where the position vector in the current configuration $\mathbf{X}$ (spatial coordinates) is related to $\mathbf{X}$, the position vector in the reference configuration (material coordinates), by $\mathbf{x}=\chi(t, \mathbf{X})$ and $\mathbf{F}$ is the deformation gradient: 


$$
\mathbf{F}=\frac{\partial \mathbf{x}}{\partial \mathbf{X}}=\frac{\partial \chi(t, \mathbf{X})}{\partial \mathbf{X}} \text { or } F_{i \alpha}=\frac{\partial x_{i}}{\partial X_{\alpha}}
$$

where the index $i$ describes the spatial coordinates, and $\alpha$ describes the material coordinates.

Using the chain rule of differentiation we can rewrite the spatial velocity gradient in terms of the material velocity $\mathbf{v}_{r}$, as follows:

$$
\frac{\partial v_{i}}{\partial x_{j}}=\frac{\partial v_{r, i}}{\partial X_{\alpha}} \cdot \frac{\partial X_{\alpha}}{\partial x_{j}} \text { or }(\nabla \mathbf{v})_{j}=\left(\nabla \mathbf{v}_{r}\right)_{i \alpha}\left(\mathbf{F}^{-1}\right)_{\alpha j}
$$

The deviatoric portion of the stress field $\boldsymbol{\sigma}_{f D}$ is then:

$$
\boldsymbol{\sigma}_{f D}=\eta\left(\nabla \mathbf{v}+(\nabla \mathbf{v})^{T}\right)=\eta\left(\nabla \mathbf{v}_{r} \mathbf{F}^{-1}+\mathbf{F}^{-T}\left(\nabla \mathbf{v}_{r}\right)^{T}\right) .
$$

Consequently, recalling that the first Piola-Kirchhoff stress tensor $\mathbf{P}$ is related to the Cauchy stress tensor by $\mathbf{P}=J \boldsymbol{\sigma} \mathbf{F}^{-T}$, the conservation of linear momentum equation reads as follows:

$$
\begin{cases}\rho_{r} \dot{\mathbf{v}}_{r}-\operatorname{Div}\left\{-J p \mathbf{I} \mathbf{F}^{-T}+\eta J\left|\nabla \mathbf{v}_{r} \mathbf{F}^{-1}+\mathbf{F}^{-T}\left(\nabla \mathbf{v}_{r}\right)^{T}\right| \mathbf{F}^{-T}\right\}=\mathbf{b} \text { for the fluid domain } \Omega_{f} \\ \rho_{r} \dot{v}_{r}-\operatorname{Div}\left(-p F^{-T}+\frac{\partial \psi(F)}{\partial F}\right)=\mathbf{b} & \text { for the solid domain } \Omega_{s} .\end{cases}
$$

In order to solve the equations for the multi-physics model using an explicit scheme, it is advantageous to treat the blood as slightly compressible. As a consequence, it is necessary to include a bulk viscosity in the definition of the Cauchy stress tensor $\sigma$ for a Newtonian incompressible fluid can be written as:

$$
\boldsymbol{\sigma}_{f}=-p \mathbf{I}+2 \eta \mathbf{D}+\lambda(\nabla \cdot \mathbf{v}) \mathbf{I}
$$

where $\eta$ is the dynamic viscosity, $\lambda$ is the bulk viscosity coefficient.

As a result, the conservation of linear momentum [10] must be rewritten accordingly, including the divergence of the velocity term. For completeness, we note that $(\nabla \cdot \mathbf{v})$ is equal to zero in case of incompressible fluid, and that takes us back to Equation [3]. 


\subsubsection{Equation of state for the fluid}

Treating arterial flow as a relatively low Mach and low pressure phenomenon, similar to sloshing in tanks, we employed a commonly used equation of state, which establishes a relationship between pressure and internal energy, the Mie-Grüneisen equation of state (Hallquist, 1999). The full expression for the Gruneisen equation defines a polynomial, known as the $\mathrm{V}_{\mathrm{s}}-\mathrm{V}_{\mathrm{p}}$ curve and can be written as:

$$
p=\frac{\rho_{r} C^{2} \mu\left[1+\left(1-\frac{\gamma_{0}}{2}\right) \mu-\frac{\alpha}{2} \mu^{2}\right]}{\left[1-\left(S_{1}-1\right) \mu-S_{2} \frac{\mu^{2}}{\mu+1}-S_{3} \frac{\mu^{3}}{(\mu+1)^{2}}\right]^{2}}+\left(\gamma_{0}+\alpha \mu\right) e
$$

where $p$ is the shock wave pressure, $e$ is the internal energy, $C$ is the intercept of the $\mathrm{V}_{\mathrm{s}}-\mathrm{V}_{\mathrm{p}}$ curve, which is the speed of sound for the medium, and $S_{1}, S_{2}$, and $S_{3}$ are the non-linear slope coefficients for the $\mathrm{V}_{\mathrm{s}}-\mathrm{V}_{\mathrm{p}}$ curve when extreme shock wave events are present, $\gamma_{0}$ is the Gruneisen gamma, and $\alpha$ is the first order volume correction to $\gamma_{0}$. The excess compression is defined by $\mu=\frac{\rho}{\rho_{r}}-1$ where $\rho$ is the current density and $\rho_{\mathrm{r}}$ is the initial density.

The implementation of the Gruneisen equation-of-state is adapted from (Gruneisen, 1926). For our purposes, we used a simplified Gruneisen equation because we assume low pressure. For the simplified equation, we only need to define $\mathrm{C}$, the speed of sound through blood, which is assumed equivalent to the speed of sound through water, i.e. $1480 \mathrm{~m} / \mathrm{s}$. By using the Gruneisen equation of state, wave propagation from secondary dynamic effects is solved as an integral part of the solution for dynamic equilibrium in an explicit analysis.

Due to the isothermal conditions, we can consider the internal energy $e$ of the system to remain constant, therefore, the simplified Grunesein equation relates pressure to the excess compression by:

$$
p=\rho_{r} C^{2} \mu+\text { const }
$$

In essence this is a linear relationship that relates pressure to the current density and can be rewritten as follows:

$$
p=C^{2} \rho+C O N S T
$$

where CONST is a constant including the value of the reference density $\rho_{r}$. 


\subsubsection{Solution method}

An explicit finite element method was used to solve the dynamic equilibrium equations in the system as implemented in LS-DYNA (LSTC, Livermore, CA). The explicit method deviates from the implicit method in the details of the equilibrium calculations. Using classical Taylor series, a central finite difference method is used to advance the position of the mesh in time, using the following equation:

$$
f^{n+1}=f^{n}+f_{, t}\left(t_{n+1 / 2}\right) \Delta t+O \Delta t^{3}{ }_{n+1 / 2}
$$

In this expression, the neglected terms are proportional to $\Delta t^{3}$ indicating that the new mesh displacement is second-order accurate in time.

Explicit time integration, while considerably more expensive than implicit time integration, is particularly suited for complex highly nonlinear systems including multiple components that experience contact.

The stability of the solution, in explicit procedures, is only guaranteed as long as the time step size is kept below a critical threshold, commonly known as the Courant condition (Woodruff, 1969). For most structural analyses, the Courant time step size is calculated by:

$$
\Delta t=\frac{0.9 L e}{\sqrt{\frac{E}{\rho}}},
$$

where $L_{e}$ represents the characteristic element length which is a function of the smallest element in the mesh, $E$ defines the elastic stiffness for that material, and finally, $\rho$ defines the density of the material for the smallest element. Note that the term in the denominator represents the speed of sound through the material. By definition, the Courant criterion ensures that the time step size is calculated based on the smallest element in the mesh and the density of that element, such that a propagating stress wave traveling at the speed of sound through the element is captured.

\subsubsection{Advection}

The ALE approach (like the pure Eulerian approach) requires that transport between elements, otherwise known as advection, be taken into account. All history variables from both the fluid and solid domains are advected; non-history variables are recalculated per step. In order to ensure stability of the numerical scheme, a split operator technique was used to solve the advection terms (Hallquist, 1999).

For each history variable $S$ (for both fluid and solid), the advected quantity $\mathrm{S}_{\mathrm{e}} \mathrm{V}_{\mathrm{e}}$ is computed using the General Advection Rule: 


$$
S_{e} V_{e}=S_{l} V_{l}+\sum_{\text {faces }} S_{l}^{j} \text { Flux } x_{j}
$$

where $\mathrm{V}_{\mathrm{e}}$ is the Eulerian element volume and $\mathrm{V}_{1}$ is the corresponding Lagrangian element volume. The simultaneous advection of state variables ensures a stronglycoupled solution to the problem.

Stability and accuracy of the advection terms further limit the time step size, in addition to the requirement arising from the Courant condition, forcing the material flux within one time step to be limited to a quarter of the element size.

\subsubsection{The material models}

Biological soft tissues are primarily composed of water and have negligible permeability and compressibility (Chuong and Fung, 1986). Carew also studied the incompressibility of the arterial wall and determined that it acts as a nearly incompressible material (Carew et al., 1968). It is also known that arteries undergo finite deformations under normal physiologic conditions. The aortic wall tissue exhibits a slightly anisotropic non-linear elastic behavior. The degree of anisotropy becomes even less prominent in the abdominal aorta in the presence of an aneurysm exhibits very little anisotropy (Vande Geest et al., 2005). For these reason, we modeled the arterial tissue as an incompressible, homogenous, hyperelastic material undergoing finite deformations. Also, experimental studies indicate that the density of the atrial wall tissue is close to that of blood, therefore, a value of $1.12 \mathrm{~g} / \mathrm{cm} 3$ was used.

In this framework, we may postulate the constitutive equations for an incompressible hyperelastic material through the following strain energy function:

$$
\psi=\widetilde{\psi}(\mathbf{F})-p(J-1)) \text { or equivalently } \psi=\hat{\psi}(\mathbf{C})-p(J-1))
$$

where the strain energy $\psi$ is defined for $J=\operatorname{det} \mathbf{F}=1$, and the scalar $p$ is the hydrostatic pressure. Furthermore, for the case of isotropy, the dependence of $\psi$ on the Cauchy-Green strain tensor $\mathbf{C}$ may be expressed by its three invariants $\mathrm{I}_{1}, \mathrm{I}_{2}, \mathrm{I}_{3}$. And since $I_{3}=1, I_{1}$ and $I_{2}$ are the two only independent variables:

$$
\psi=\psi\left(I_{1}, I_{2}\right)
$$

Based on experimental studies, a polynomial strain energy function was chosen for the abdominal aorta, as follows:

$$
\psi_{\text {AORTA }}=a_{1}\left(I_{1}-3\right)+a_{2}\left(I_{1}-3\right)^{2},
$$

where $a_{1}$ and $a_{2}$ are the material parameters. 
For simplicity we have utilized the same form of the strain energy function for the healthy aorta and for the aneurismal aorta. Coefficients for the latter were obtained from the study of Raghavan et al., performed on AAA specimen obtained during surgery (Raghavan and Vorp, 2000). For healthy tissue, coefficients were obtained by regression analysis from experimental data on aortic specimens from autopsies for patients aged fifty and over (Vande Geest et al., 2004). The coefficients were $a_{1}=13.9 \mathrm{~N} / \mathrm{cm}^{2}$ and $a_{2}=150 \mathrm{~N} / \mathrm{cm}^{2}$, and $a_{1}=17.4 \mathrm{~N} / \mathrm{cm}^{2}$ and $a_{2}=188.1 \mathrm{~N} / \mathrm{cm}^{2}$, respectively, for the healthy aortic wall portion and the aneurysmal wall.

Treating the thrombus as slightly denser than blood but not as dense as the arterial wall, a density of $1.10 \mathrm{~g} / \mathrm{cm}^{3}$ was prescribed. The hyperelastic material relationship for the ILT was used as previously published with parameters $c_{1}$ and $c_{2}$ for the strain energy function reported hereafter equaling $2.81 \mathrm{~N} / \mathrm{cm}^{2}$ and $2.86 \mathrm{~N} / \mathrm{cm}^{2}$, respectively (Wang et al., 2001):

$$
\psi_{I L T}=c_{1}\left(I_{2}-3\right)+c_{2}\left(I_{2}-3\right)^{2},
$$

where $c_{1}$ and $c_{2}$ are the material parameters

Recently, Brown et al. (2003) experimentally measured the in-vivo mechanical behavior of porcine internal organs. From this paper, stress-strain behavior plots for the bladder, gallbladder, large intestine, liver, small intestine, spleen, and stomach are available. As a first attempt, we averaged the small and large intestine characteristics and applied them to the abdominal cavity component in our model. Similar properties were assigned to the peritoneum tissue. The constitutive model used for the abdominal cavity component is a fully-recoverable low-density foam (Hallquist, 1999). This foam model is mainly used for modeling highly compressible low density foams, and is well suited for applications that require that the foam returns to its original shape once the load is removed. No hysteretic unloading per cardiac cycle was assumed. As a first approximation, the density of the foam is defined as $1.15 \mathrm{~g} / \mathrm{cm}^{3}$ assuming the intestinal content is slightly denser than the aorta.

The blood is defined as a Newtonian fluid with a density of $1.06 \mathrm{~g} / \mathrm{cm}^{3}$, and a viscosity of 0.00319 Pa-s (Fung, 1993). Blood is essentially a suspension of erythrocytes in plasma and shows anomalous viscous properties at low velocities and small diameters. However, the Newtonian assumption has been considered acceptable since minor differences in the basic flow characteristics are introduced through the non-Newtonian hypothesis for large arteries. Moreover, especially when the velocities are low, the wall shear stresses are several orders of magnitude lower than the stress due to the pressure acting on the wall; consequently, the overall wall stress is minimally influenced by the non-Newtonian behavior.

Since the spinal column is assumed orders of magnitude stiffer than any other component in the model, and its sole purpose is to provide realistic support through contact to the aorta, a rigid material is used. Even though rigid, realistic material properties were used as they play a significant role in calculating penalty stiffness 
forces in the contact definition, namely, an elastic modulus of $18 \mathrm{GPa}$ and a density of $2.20 \mathrm{~g} / \mathrm{cm}^{3}$ (Reilly et al., 1975).

\subsubsection{Boundary and loading conditions}

The appropriate boundary and loading conditions were defined on all considered domains, namely, from the outside to the inside (see Figure 2):

- abdominal cavity component (solid);

- spine (solid - rigid);

- retroperitoneum membrane (solid - shell);

- soft tissue (solid);

- wall (solid - shell);

- thrombus (solid);

- lumen (fluid).

as well as on all boundaries:

- outer surface of the spine column (rigid);

- upper boundary surface of the aorta, thrombus and fluid;

- lower boundary surface of the aorta, thrombus and fluid;

- outer surface of the abdominal intestinal component;

- surface interface between spine column and retroperitoneum membrane (bilateral contact);

- surface interface between retroperitoneum membrane and soft tissue (tied contact);

- interface between soft tissue and outer surface of the aorta (bilateral contact);

- interface between the inner surface of the aorta and the thrombus outer surface (tied contact);

- interface between the inner surface of the thrombus component and the fluid (ALE - the fluid mesh is remeshed at each step in order to follow the deformation of the solid mesh).

For completeness, it is worth recalling that the reference used for the computational simulation is Lagrangian, therefore, if we consider an oriented infinitesimal surface $\mathbf{n} d \Sigma$ represented in the Eulerian coordinate system, the corresponding quantity for a Lagrangian observer is:

$$
\mathbf{n} d \Sigma=J \mathbf{n}_{r} \mathbf{F}^{-1} d \Sigma,
$$

where $\mathbf{n}$ is the normal to the surface in the current configuration and $\mathbf{n}_{\mathrm{r}}$ is the normal to the surface in the reference configuration.

The boundary and loading conditions are summarized in the following. 
The spinal column is constrained in all translational and rotational degrees of freedom. The presence of the spine as a geometric entity aids in the anatomical understanding of the model and, moreover, provides realistic contact conditions to the expanding aorta that would not have been achieved by simply constraining all degrees of freedom in the posterior surface of the aortic wall. The bottom of the aorta is constrained in the vertical direction, to mimic tethering, but is free to develop radial dilation with the passing of each pulse. The upper boundary of the aorta segment is constrained in all translational degrees of freedom. Lastly, the anterior face of the intestinal component, furthest away from the vessel, is also constrained in all translational and rotational degrees of freedom.

At the inflow boundary, a parabolic velocity field is prescribed in normal direction through an ambient reservoir (Figure 4). The ambient reservoir is assumed to have an infinite supply of fluid. The velocity boundary conditions imposed are based on flow rate signals in the descending aorta as reported by Mills (Mills et al., 1970), scaled to obtain a time period of $1 \mathrm{sec}$ and a realistic average peak-systolic Reynolds number (of approximately 1000 ). At the outflow the fluid is considered stress-free and at the inner thrombus-fluid interface there is a no slip condition. A time-dependent isobaric pressure is imposed at the outlet. At the beginning of the simulation the fluid is quiescent and twelve cardiac cycles have been simulated to achieve a solution independent from the initial conditions.

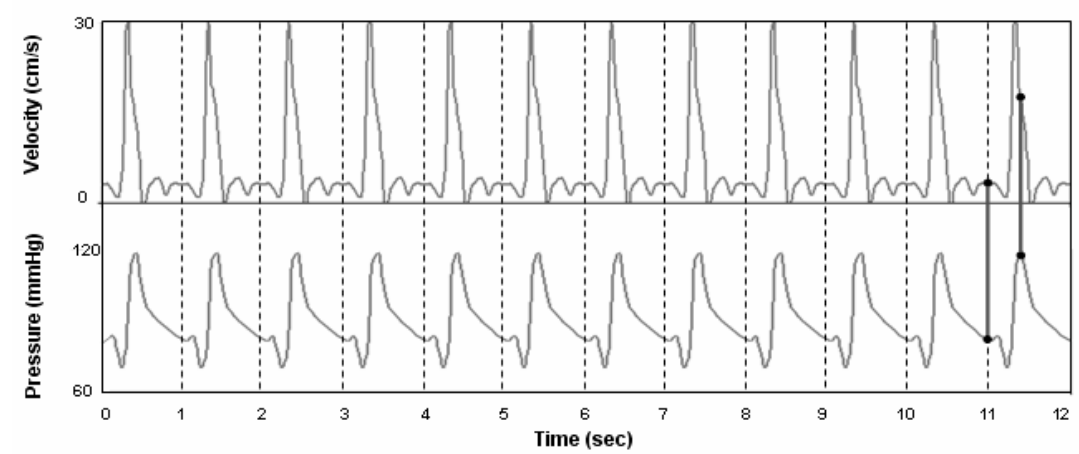

Figure 4. Velocity and pressure loading conditions

The restraint from the spinal column to the soft tissue covering the aorta is defined through an automatic-surface-to-surface contact algorithm which transmits bilateral, nonlinear contact forces at the interface. This contact definition serves two purposes: first, it provides the necessary link between the aorta and spinal column where interface pressures are naturally generated thus pressing the aorta between the spine and abdominal cavity contents, and secondly, it tracks these contact forces on the retro peritoneum making it possible to quantify and document interaction forces and stresses. 


\section{Results}

The analysis was executed on a Hewlett-Packard C8000 UNIX workstation with four processors and two gigabyte of s-RAM each. The computational time required to complete twelve cardiac cycles was approximately forty-eight hours. Due to the vast amount of spatial and temporal results data made available by an explicit dynamic analysis, we arbitrarily begin by examining the results from the inside of the model and then work our way towards the outer components. The first set of results examined will be the fluid behavior within the lumen channel since the fluid flow is the driving force for the analysis, to ensure dynamic steady state has been reached. Then we will focus our attention on the ILT response to the flow, followed by the vessel response to fluid and ILT, and finally the abdominal cavity component.

\subsection{Qualitative results}

Figure 5a shows a viewing plane midway down the healthy aorta segment. Within this plane, the diameter change as a function of time was plotted, shown in Figure $5 \mathrm{~b}$. The FEA results show a stabilization of pulsatile diameter increase in the vicinity of $13 \%$, which is compatible with the percentage increase per cardiac cycle that we measured by means of dynamic MRI on healthy volunteers (approximately 10 to $14 \%$ over the native diameter).

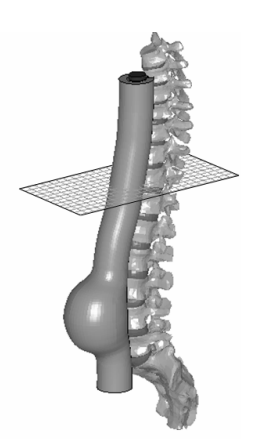

a)

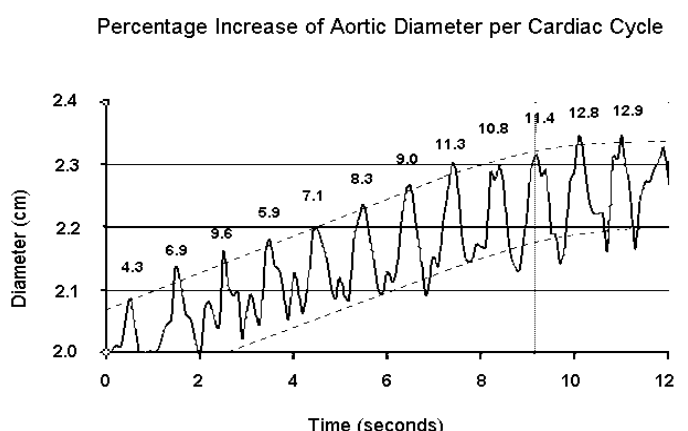

b)

Figure 5. Percentage excursion aortic diameter over time

Figure 6 shows the fringe plots of velocity magnitude along the entire length of the vessel, plotted every four seconds. An arbitrary fringe scale of zero to $10 \mathrm{~cm} / \mathrm{s}$ was assigned to the fluid domain to assist in the visualization of the fluid motion; blue denoting zero or slow velocity, and red denoting velocities equal to or greater than $10 \mathrm{~cm} / \mathrm{s}$. At twelve seconds, all of the blood contained inside the healthy aorta 
and aneurysmal sac is in motion, driven by the reservoir at the top of the lumen channel and its own momentum.

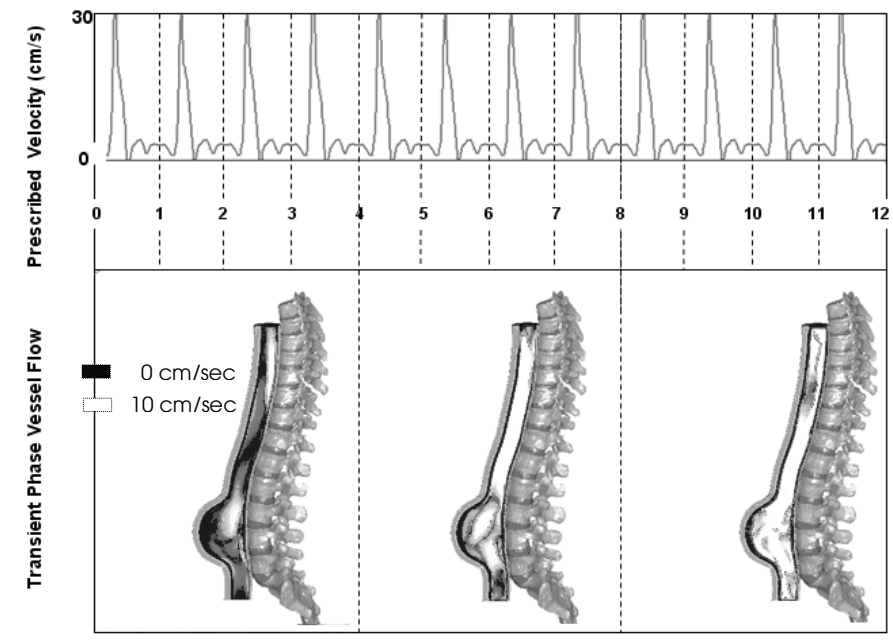

Figure 6. Velocity magnitude along the length of the vessel $(\mathrm{cm} / \mathrm{sec}$.)

One advantage of simulating a tight coupling between the solid and fluid domains is that the Windkessel Effect can be captured. The Windkessel effect states that part of the elastic energy stored in the aortic wall at each cardiac cycle is returned as kinetic energy to the blood during each diastolic phase, allowing the aorta to act as an auxiliary pump. Figure 7 shows a visualization of the Windkessel effect: the wall elastic energy peaks (light grey curve) are followed by the blood's kinetic energy (dark grey curve) peaks for the time interval between 0 and 11 seconds of simulation. Beyond 11 seconds of simulation there is the appearance of flow disturbance in the sac, as will be discussed in the next paragraph.

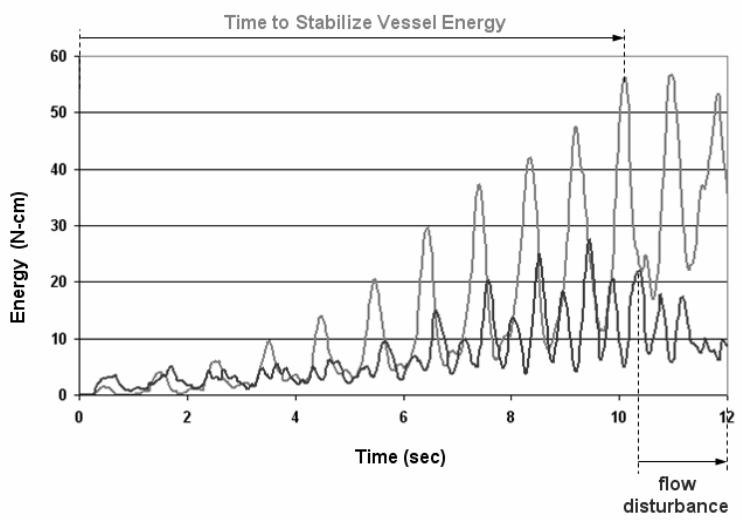

Figure 7. Visualization of the Windkessel Effect over the length of the simulation 
Figure 8a shows a viewing plane positioned mid-way through the sac where cross-sectional deformations are shown; Figure $8 \mathrm{~b}$ shows the deformed configuration at time zero, 11.0 and 11.4 seconds of simulation. Larger displacements occur towards the right posterior quadrant, as is to be expected due to the asymmetry of the model.
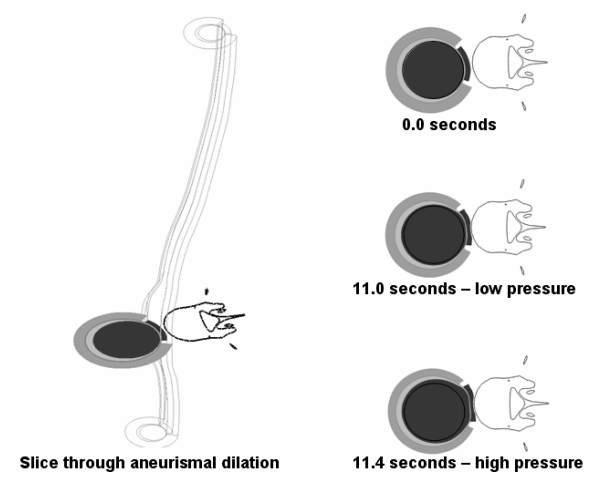

Figure 8. Cross-sectional deformation at the level of maximum dilatation in the $A A A$

To evaluate the evolved flow patterns within the aneurysmal sac after the eleventh cardiac cycle, plots of instantaneous flow trajectories were collected, as shown in Figure 9. Within the $11^{\text {th }}$ cycle, results indicate lowest pressure inside the aneurysmal sac at 11.0 seconds, and highest pressure within the sac at 11.4 seconds. Accordingly, subsequent results will be collected at those times. Figure 9a shows the sagittal plane velocity trajectories at 11.0 seconds, while Figure $9 \mathrm{~b}$ shows the equivalent plot at 11.4 seconds. For this figure, an arbitrary range for velocity was set from zero to $12 \mathrm{~cm} / \mathrm{s}$ which maximizes the visual differences between the two time states.

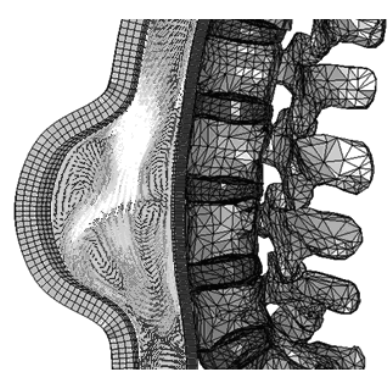

a
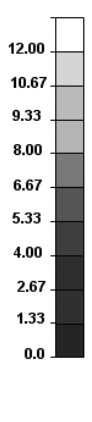

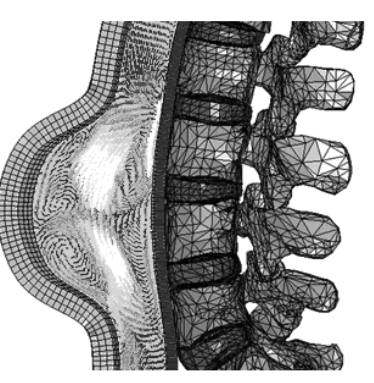

b

Figure 9. Fluid velocity trajectories in the aneurismal sac (cm/sec.); a) $11.0 \mathrm{sec}$., b) $11.4 \mathrm{sec}$. of simulation 


\subsection{Quantitative results}

We will now turn the attention to quantitative measures of aortic mechanics behavior. Figures $10 \mathrm{a}$ and $\mathrm{b}$ show the maximum principal stress distribution within the body of the ILT at times 11.0 and 11.4 seconds, respectively. An arbitrary fringe scale of 0 to $2 \mathrm{~N} / \mathrm{cm}^{2}$ is assigned to aid in clear visualization of the stress distribution. The peak thrombus stress within the AAA sac is approximately $2.45 \mathrm{~N} / \mathrm{cm}^{2}$ which occurs at 11.4 seconds, and is in good agreement with published data (Wang et al., 2002; Di Martino et al., 2003).

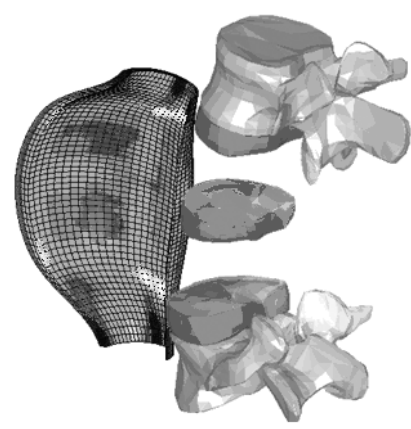

a)
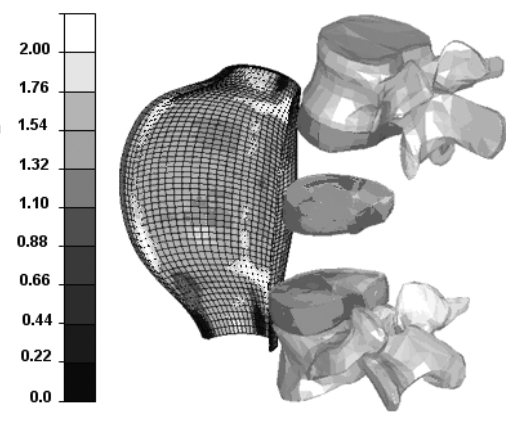

b)

Figure 10. Maximum principal stresses $\left(N / \mathrm{cm}^{2}\right)$ in a longitudinal section of the intraluminal thrombus; a) $11.0 \mathrm{sec}$., b) $11.4 \mathrm{sec}$. of simulation

Figure 11 shows the maximum principal stress distributions in both the healthy and aneurismal aorta. To aid in visualization, an arbitrary stress range of $10 \mathrm{~N} / \mathrm{cm}^{2}$ to $35 \mathrm{~N} / \mathrm{cm}^{2}$ was assigned. The maximum stress in the aortic segment at 11.0 seconds is $44.7 \mathrm{~N} / \mathrm{cm}^{2}$, and is located along the length of the healthy segment in the posterior region. The location of the maximum stress is influenced by the flow impingements along the posterior vessel surface caused by the vessel curvature along with restraint from the spinal column. At the same time instant the stress in the anterior region of the healthy aortic segment is approximately $15 \mathrm{~N} / \mathrm{cm}^{2}$, which is consistent to published value for the normal aortic tract when the curvature of the vessel is not included in the model (Raghavan et al., 2000). Referring to Figure 11b, the peak wall stress for the aneurismal segment is located posterior next to the spinal column. The peak wall stress at 11.4 seconds is $36.6 \mathrm{~N} / \mathrm{cm}^{2}$, which is within the range of published peak stress values (Raghavan et al., 2000; Wang et al., 2002).

Figure 12 illustrates information about the vessel wall that has not been previously documented, the vessel thinning as a function of time. The percentage of thinning between 11 and 12 seconds of simulation is shown for four different locations on the aortic wall, shown in the left panel of the figure. 


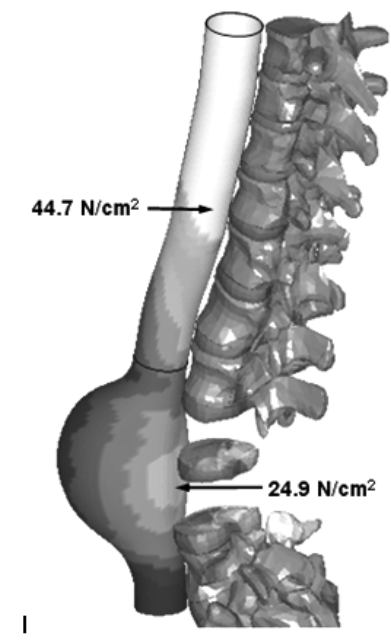

a)

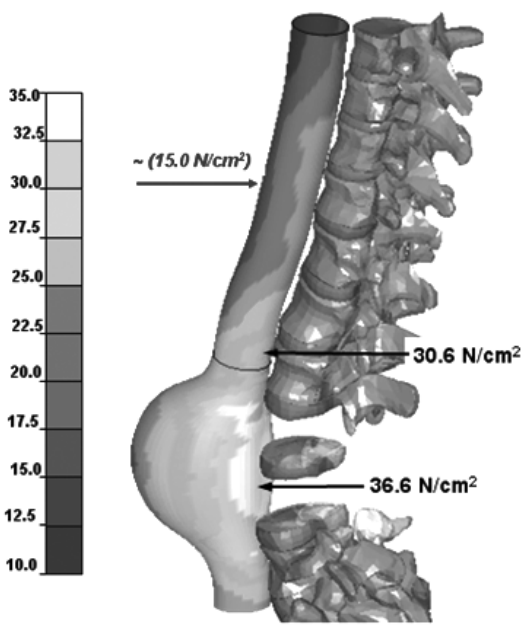

b)

Figure 11. Maximum principal stresses on the aortic wall at two instants in the cardiac cycle $\left(\mathrm{N} / \mathrm{cm}^{2}\right)$; a) $11.0 \mathrm{sec}$., b) $11.4 \mathrm{sec}$. of simulation

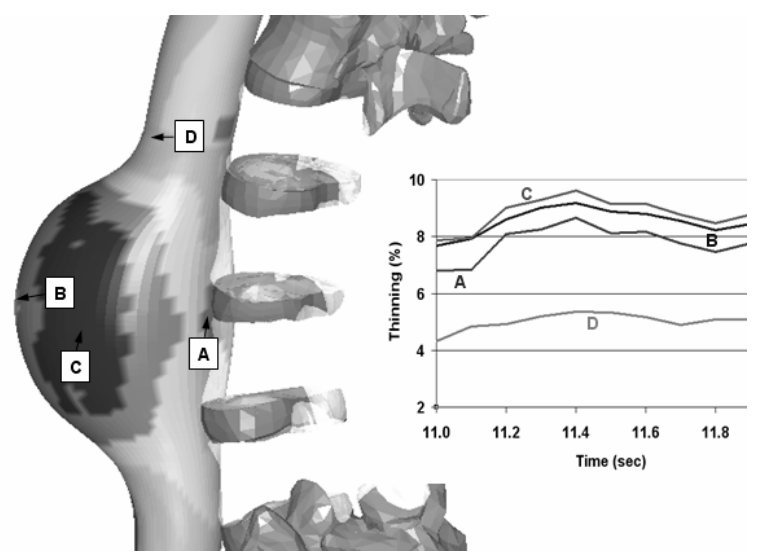

Figure 12. Vessel wall thinning versus time at 4 different locations on the model (shown on the left)

Lastly, Figure 13 shows the compressive stress distribution through the thickness of the intestinal component. Following De St. Venant's principle, the thickness has to be deep enough so that the translational and rotational constraints prescribed on the anterior face of the foam, furthest away from the vessel, do not influence the vessel behavior. The compressive stress distribution shown in Figure 13 indicates a sufficient thickness as elevated stresses do not reach the outer surface. 


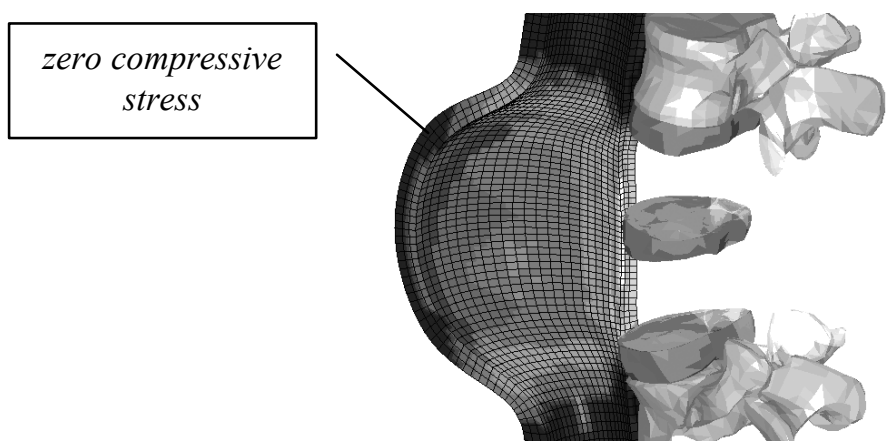

Figure 13. Compressive stress profile through the thickness of the abdominal cavity component (dark grey indicates zero compressive stress)

\section{Discussion}

The current study presents a strongly-coupled, dynamic, fluid structure interaction simulation of a long segment of aorta including aneurysm. All major features of the biological system of AAA were represented, including nonlinear elastic, non-homogeneous constitutive material properties, pulsatile loading due to the blood flow, and anatomically correct boundary conditions along the length of the aorta, exemplified by the spinal column and abdominal cavity contents.

We treated the interaction between the systemic circulation and the vessel that supports the flow as the fundamental forcing function acting on the deformable wall and causing time-varying wall dynamics. Our method underscores the fact that the stresses that develop in-vivo within the artery wall are strongly dependent on the timing (phase) during the flow cycle.

The results demonstrate a percentage excursion of the aortic diameter during each pulsatile dilatation that stabilizes in the vicinity of physiological values (thirteen \%) (Figure 5). This result corroborates the assumptions made regarding the abdominal cavity component, which plays an important role in achieving a correct dilation of the aorta.

The Windkessel effect, which states that the ventricular ejection is complemented by the effect of the elastic arteries (the aorta) that provide an auxiliary pumping effect by storing elastic energy during systole and then transferring it to the blood in the form of kinetic energy during the cardiac diastolic phase, is clearly documented in our results. As the vessel wall stores energy, the wall elastic energy peaks (light grey curve in Figure 7), and then releases the stored energy causing a drop in the energy curve in the diagram. An increase in the blood's kinetic energy follows, indicating that the vessel wall is aiding the blood flow downstream. This trend is visible from 6 to 11 seconds where fluid momentum is accumulating and then stabilizing around 11 seconds. Worth noting, there is a 
sudden decline in the kinetic energy curve after 11 seconds. This observance can be explained by monitoring the complex flow patterns that emerge within the aneurysmal sac creating a retrograde flow back into the healthy, proximal extension as shown in the right hand view of Figure 9. This cyclic backflow has the effect of congesting flow and reducing the average fluid velocity at the entrance of the sac, which causes a decrease in kinetic energy.

Turning the attention to quantifiable localized results, we compared our results with data reported by Wang et al., and to our previous results. Both models included the presence of the ILT and were solved applying a static pressure load to the inner surface of the vessel corresponding to an average systolic peak pressure (Wang et al., 2002; Di Martino and Vorp, 2003). The maximum principal stresses distribution and values within the body of the ILT obtained in the current analysis were compatible with those previously reported; maximum principal stress were $2.45 \mathrm{~N} / \mathrm{cm}^{2}, 6 \mathrm{~N} / \mathrm{cm}^{2}$, and $2.5 \mathrm{~N} / \mathrm{cm}^{2}$ on average, respectively, in the current study, in Wang's study and in our previous static study performed on hypothetical aneurysm geometries (Wang et al., 2002; Di Martino and Vorp 2003). These values are well within the margins of ultimate strength for ILT, which is approximately $30 \mathrm{~N} / \mathrm{cm}^{2}$ (Wang et al., 2001).

The stress results obtained for the aortic wall in the area of the aneurysm dilatation are also in accord with published data; maximum principal wall stress for our study was $36.6 \mathrm{~N} / \mathrm{cm}^{2}$ and $30 \mathrm{~N} / \mathrm{cm}^{2}$ on average in the study by Wang et al.,(Wang et al., 2002). It is interesting to note that in the area of the healthy descending aorta, we found maximum principal stress values higher than those in the aneurysm area; Figure 12a shows a maximum principal stress value of $44.7 \mathrm{~N} / \mathrm{cm}^{2}$ acting along the posterior wall the vessel. This result deserves a careful examination. The presented study provides the first computational results obtained from a strongly-coupled fluid structure interaction analysis including most of the descending aorta. We included the effect of curvature of the spine and we made assumptions on the thickness of the vessel wall, based on previously reported experimental data. The higher stress value for the healthier portion of the aorta is a result of the flow impingements on the curved aortic wall, due to the spinal curvature. The aneurysmal portion of the aorta, on the other hand, includes no calcifications or local thinning of the wall, which could definitely lead to higher local stress values. Nevertheless, it is worth noting that the reported strength of the healthy aortic specimen is approximately twofold, on average, of that of aneurysm specimens (Vorp et al., 1996). As a consequence, the aneurysmal aorta, despite the lower absolute stress, falls within a narrower margin of reserve strength than the healthy aortic region. Our results may also indicate that tortuosity in the aneurysmal aorta may impact not only flow conditions but also the vessel wall stress levels, indicating a higher risk of rupture for tortuous aneurysms. When the thrust of the flow activity is in the aneurismal sac, at 11.4 seconds, the wall stress at the same location in the healthy aorta is $15 \mathrm{~N} / \mathrm{cm}^{2}$, which correlates with previously reported results in a straight aortic model (Vorp et al., 1998). 
The simulation provided accurate time-varying wall thinning measurements throughout the cardiac cycle (Figure 13). This piece of information may be very useful to assist in the design and evaluation of attachment site mechanisms for endovascular prosthesis. Endovascular devices in fact often rely on hooks penetrating the aortic wall to fix the prosthesis in place and may experience detachments due to unexpected thinning and improper design.

There are several limitations to this FEA model. No branches from the main artery have been considered, including the renal arteries. We postulated that since the descending aorta contains the largest volume of fully-developed flow, deceleration at the renal bifurcation prior to the AAA sac can be neglected. Also, the geometric model does not include the iliac bifurcation distal to the sac. The material model for the aneurysmal and healthy aortic regions has been assumed to be non linearly elastic and isotropic neglecting anisotropy and viscoelastic properties of the wall tissue. The material properties for the abdominal cavity component have been obtained from porcine tests as the corresponding material properties for humans have not been documented. The retro peritoneous membrane has been assumed to have the same characteristics as the abdominal cavity component.

Although the mesh size and element types were chosen on the basis of previous successful mesh independency analysis (Di Martino et al., 2003) a thorough mesh sensitivity analysis on this computational model is necessary to determine whether or not the chosen element size, distribution, and mathematical formulations are optimal for this application. Another limitation of the analysis is represented by the absence of an appropriate after-load at the outflow. Inherent in the complexity of simulating the in-vivo flow conditions of a closed system using an open system model are challenges with boundary assumptions. As such, boundary condition details at the bottom of the vessel are on on-going area of exploration in order to stabilize and maintain the fluid kinetic energy indefinitely.

As this explicit methodology evolves, each of these concerns will be addressed fully.

\section{Conclusion}

To date, there appear to be no published analyses of a strongly-coupled fluid structure interaction analysis on a full-length descending aorta and surrounding anatomical components, solved using explicit finite element methods. We have shown that, using an explicit solution scheme, all principal features of the AAA system were captured, including fluid structure interaction between fluid and solid domain, contact with the spine and the retroperitoneum membrane and the effects of the abdominal organs.

Extreme AAA tortuosity, realistic iliac bifurcations, and cardiac applications including ventricles and atria, are all examples of potential applications where a 
strongly-coupled fluid structure interaction solution methodology may prove essential to depict the actual in-vivo mechanics.

In conclusion, we believe that the methodology presented provides the foundation for exploring computational cardiovascular dynamic simulations within the explicit regime. Further developments will address the limitations identified and extend our range of applications to other cardiovascular areas.

\section{Acknowledgements}

The authors would like to thank Dr. Ender Finol and Ms. Christine Scotti for their helpful comments and suggestions, and Dr. Vande Geest for the experimental data on the aortic wall. This work is supported in part by the Pennsylvania Infrastructure Technology Alliance, a partnership of Carnegie Mellon, Lehigh University, and the Commonwealth of Pennsylvania's Department of Community and Economic Development (DCED), and by the National Institutes of Health grant R01 HL79313-01A1.

\section{References}

Ailawadi G., Eliason J. L., Upchurch G. R. Jr., "Current concepts in the pathogenesis of abdominal aortic aneurysm”, J Vasc Surg., vol. 38, n 3, 2003, p. 584-588.

Bluestein D., Niu L., Schoephoerster R. T., Dewanjee M. K., "Steady flow in an aneurysm model: correlation between fluid dynamics and blood platelet deposition", J Biomech Eng, vol. 118, n 3, 1996, p. 280-286.

Brown J., Rosen J., Sinanan M., Hannaford B., "In-vivo and postmortem compressive properties of porcine abdominal organs", Lecture notes in Computer Science, vol. 2878, 2003, p. 238-245.

Carew T. E., Vaishnav R. N., D.J. P., "Compressibility of the arterial wall", Circulation Research, vol. 23, 1968, p. 61-68.

Chuong C. J., Fung Y. C., "Compressibility and constitutive equation of arterial wall in radial compression experiments", J Biomech, vol. 17, n 1, 1984, p. 35-40.

Curci J. A., Liao S., Huffman M. D., Shapiro S. D., Thompson R. W., "Expression and localization of macrophage elastase (matrix metalloproteinase-12) in abdominal aortic aneurysms", J Clin Invest, vol. 102, n 11, 1998, p. 1900-1910.

Di Martino E., Mantero S., Inzoli F., Melissano G., Astore D., Chiesa R., Fumero R., "Biomechanics of abdominal aortic aneurysm in the presence of endoluminal thrombus: experimental characterisation and structural static computational analysis", European Journal of Vascular \& Endovascular Surgery, vol. 15, n 4, 1998, p. 290-299.

Di Martino E. S., Guadagni G., Fumero A., Ballerini G., Spirito R., Biglioli P. and Redaelli, A., "Fluid-structure interaction within realistic three-dimensional models of the 
aneurysmatic aorta as a guidance to assess the risk of rupture of the aneurysm", Medical Engineering \& Physics, vol. 23, $\mathrm{n}^{\circ}$ 9, 2001, p. 647-655.

Di Martino E. S., Vorp D. A., "Effect of variation in intraluminal thrombus constitutive properties on abdominal aortic aneurysm wall stress", Ann Biomed Eng, vol. 31, n 7 , 2003, p. 804-809.

Di Martino E. S., Yamakawa S., Shimada K. and Vorp D., "Requirements for mesh size and mesh type in non linear computational finite element method structural analyses", Second MIT Conference on Computational Fluid and Solid Mechanics, Cambridge, MA, 2003, Cambridge, MA, MIT.

Fillinger M. F., Raghavan M. L., Marra S. P., Cronenwett J. L. and Kennedy F. E., "In vivo analysis of mechanical wall stress and abdominal aortic aneurysm rupture risk", Journal of Vascular Surgery, vol. 36, n 3, 2002, p. 589-597.

Fung Y. C., Biomechanics-Mechanical Properties of Living tissue, Springer, 1993.

Gruneisen E., Handbuck der Physic, vol. 10, n² 22, Berlin, Springer, 1926.

Hallquist J., LS-DYNA Theoretical manual, Livermore, CA, Livermore Software Corporation, 1999.

Harter L. P., "Ultrasonic evaluation of abdominal aortic thrombus", J. Ultra. Med, vol. 1, 1982, p. 315-318.

Huffman M. D., Curci J. A., Moore G., Kerns D. B., Starcher B. C., Thompson R. W., "Functional importance of connective tissue repair during the development of experimental abdominal aortic aneurysms”, Surgery, vol. 128, n 3, 2000, p. 429-38.

Mills C., Gabe I., Gault J., Mason D., Ross J. J., Brunwald E. and Shillingford J., "Pressureflow Relationships and Vascular Impedance in Man”, Cardiovasc. Res., vol. 4, 1970, p. $405-417$.

Mower W. R., Quinones W. J. and Gambhir S. S., "Effect of intraluminal thrombus on abdominal aortic aneurysm wall stress", J Vas Surg, vol. 26, 1997, p. 602-608.

Nakamura N., Wada S., Yokosawa S., Isoda H., Tsubota K. and Yamaguchi T., "Flow in an integrated model of the heart and aorta", XXI Ineternational Conference of Theoretical and Applied Mechanics, Warsaw, 2004. Springer Verlag.

Peattie R. A., Riehle T. J., Bluth E. I., "Pulsatile flow in fusiform models of abdoiminal aortic aneurysms: flow fields, velocity patterns and flow-induced wall stresses", J Biomech Eng, vol. 126, n 4, 2004, p. 438-446.

Raghavan M. L., Vorp D. A., "Toward a biomechanical tool to evaluate rupture potential of abdominal aortic aneurysm: identification of a finite strain constitutive model and evaluation of its applicability", Journal of Biomechanics, vol. 33, 2000, p. 475-482.

Raghavan M. L., Vorp D. A., Federle M. P., Makaroun M. S. and Webster M. W., "Wall stress distribution on three-dimensionally reconstructed models of human abdominal aortic aneurysm", Journal of Vascular Surgery, vol. 31, n 4, 2000, p. 760-769. 
Redaelli A., Rizzo G., Arrigoni S., Di Martino E., Origgi D., Fazio F. and Montevecchi F., "An assisted automated procedure for vessel geometry reconstruction and hemodynamic simulations from clinical imaging", Comput Med Imaging Graph, vol. 26, n 3, 2002, p. 143-52.

Reilly D. T. and Burstein A. H., "The elastic and ultimate properties of compact bone tissue", Journal of Biomechanics, vol. 8, 1975, p. 393-405.

Schmid P., Stuber M., Boesiger P., Hess O. M. and Niederer P., "Determination of displacement, stress- and strain-distribution in the human heart: a FE-model on the basis of MR imaging”, Technol Health Care, vol. 3, n³, 1995, p. 209-214.

Smith D., Sacks M., Vorp D. and Thornton M., "Surface geometric analysis of anatomic structures using biquintic finite element interpolation", Ann Biomed Eng, vol. 28, n 6 , 2000, p. 598-611.

Steinman D. A., Vorp D. A. and Ethier C. R., "Computational modeling of arterial biomechanics: insights into pathogenesis and treatment of vascular disease", Journal of Vascular Surgery, vol. 37, ${ }^{\circ}$ 5, 2003, p. 1118-1128.

Vande Geest J. P., Sacks M. S. and Vorp D. A., "Age dependency of the biaxial biomechanical behavior of human abdominal aorta", $J$ Biomech Eng, vol. 126, n 6 , 2004, p. 815-822.

Vande Geest J. P., Sacks M. S. and Vorp D. A., "The effects of aneurysm on the biaxial mechanical behavior of human abdominal aorta", J Biomech, in press 2005 (available on-line).

Vorp D. A., Raghavan M. L., Muluk S. C., Makaroun M. S., Steed D. L., Webster M W., "Wall strength and stiffness of aneurysmal and nonaneurysmal abdominal aorta", Ann. NY Acad. Sci, vol. 800, 1996, p. 274-277.

Vorp D. A., Raghavan M. L. and Webster M. W., "Mechanical wall stress in abdominal aortic aneurysm: Influence of diameter and asymmetry", Journal of Vascular Surgery, vol. 27, n 4, 1998, p. 27.

Wang D. H., Makaroun M., Webster M. W., Vorp, D. A., "Mechanical properties and microstructure of intraluminal thrombus from abdominal aortic aneurysm", Journal of Biomechanical Engineering, vol. 123, $\mathrm{n}^{\circ}$ 6, 2001, p. 536-539.

Wang D. H., Makaroun M. S., Webster M. W. and Vorp D. A., "Effect of intraluminal thrombus on wall stress in patient-specific models of abdominal aortic aneurysm", Journal of Vascular Surgery, vol. 36, n 3, 2002, p. 598-604.

Wolters B. J., Rutten M. C., Schurink G. W., Kose U., de Hart J., van de Vosse F. N., “A patient-specific computational model of fluid-structure interaction in abdominal aortic aneurysms", Med Eng Phys, vol. 27, n 10, 2005, p. 871-883.

Woodruff L. W., University of California Report UCRL 50621, 1969. 
